\title{
An apparent symmetry property of the mean velocity gradient in turbulent Poiseuille flows and its implications
}

\author{
F. Laadhari $\left.{ }^{a}\right)$ \\ Laboratoire de Mécanique des Fluides et d'Acoustique, Université de Lyon, Université Claude Bernard Lyon 1, \\ Ecole Centrale de Lyon, INSA de Lyon, CNRS UMR 5509, 36 Avenue Guy de Collongue, \\ F-69134 Ecully, France
}

(Received 20 July 2011; accepted 10 October 2011; published online 31 October 2011)

\begin{abstract}
A novel feature of the mean velocity gradient in turbulent parallel Poiseuille flows has been found using the results available in databases of direct numerical simulations at moderately high friction Reynolds number $R_{\tau}$, up to 2000 . The computed turbulence statistics show that the logarithm of the mean velocity gradient, normalized by its value at the quarter-channel height, is very close to symmetric with respect to this position. At this location, the ratio of the viscous transport term to the viscous stress is a minimum. The range of validity of this property increases with the Reynolds number and is between $y / h=0.1$ and $y / h=0.9$ for $R_{\tau}=2000$. This property is a convenient tool in channel flow analysis since the velocity profile in the wall region can be accurately predicted from values much further away. We explore in some detail the properties that follow from this discovery. (C) 2011 American Institute of Physics. [doi:10.1063/1.3657819]
\end{abstract}

Direct numerical simulations (DNS) of turbulent flows have become an indispensable tool for studying the statistical properties and structural characteristics of turbulence and more particularly of wall bounded turbulent flows. Available DNS databases for wall-bounded flows include channel, pipe, and boundary layer flows with friction Reynolds number or Kármán number $R_{\tau}=u_{\tau} h / \nu$ up to 2000 ( $u_{\tau}$ is the friction velocity, $h$ the channel half-width, and $\nu$ the kinematic viscosity). The aim of this letter is to investigate, using DNS databases, the evolution of the mean velocity gradient in fully developed stationary incompressible turbulent Poiseuille flows.

For these flows, the normalized mean streamwise momentum equation reduces to

$$
-\frac{\mathrm{d} \overline{u v}^{+}}{\mathrm{d} y^{+}}+\frac{\mathrm{d} S^{+}}{\mathrm{d} y^{+}}=-\frac{1}{h^{+}},
$$

where $S$ is the wall-normal gradient of the mean streamwise velocity $\mathrm{d} \bar{U} / \mathrm{d} y, \overline{u v}$ the mean Reynolds shear-stress, and $y$ the wall-normal coordinate. The superscript $\left({ }^{+}\right)$indicates normalization with the wall variables, $\nu$ and $u_{\tau}$. Equation (1) represents the equilibrium between the mean pressure gradient and the sum of the viscous and Reynolds stress gradients. The integration of Eq. (1) with appropriate boundary conditions yields the linear variation of the total mean stress with the wall-normal distance

$$
-\overline{u v}^{+}(\eta)+S^{+}(\eta)=1-\eta
$$

with $\eta=y / h$. Under transformation from a Cartesian to a cylindrical coordinate system, Eq. (2) can also be shown to hold for fully developed turbulent flow in a pipe of radius $R$ with $\eta=y / R$.

Figure 1 shows the profiles of the logarithm of $S(\eta)$ and $S(1-\eta)$, normalized by $S(0.5)$, for channel and pipe flows. The channel data are from the DNS results of Jiménez and

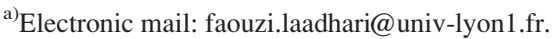

Hoyas $^{1}$ for the three Kármán numbers $R_{\tau}=h^{+}$of 550, 940, and 2000, and the data for pipe flow are from the DNS results of $\mathrm{Wu}$ and Moin. ${ }^{2} \mathrm{We}$ present, here, a hitherto unreported symmetry in the logarithm of the mean velocity gradient, $S$, which can be characterized by the following relation:

$$
\log \frac{S(\eta)}{S(0.5)} \simeq-\log \frac{S(1-\eta)}{S(0.5)}
$$

or equivalently

$$
\frac{S(\eta)}{S(0.5)} \simeq \frac{S(0.5)}{S(1-\eta)} .
$$

The apparent symmetry around $\eta \approx 0.5$, the channel quarter-width, is a novel and surprising feature and appears to be generally respected. The DNS data conform well with this symmetry property over a wide range of wall-normal positions, whose extent increases with increasing Reynolds number. The lower limit of the validity range of the symmetry property is about 150 wall-units, while the upper limit increases with the Reynolds number and is approximately equal to $\eta \approx 0.8,0.85$, and 0.9 for $R_{\tau}=550,940$, and 2000 , respectively. In these ranges, the relative difference observed between the two terms is, respectively, less than $0.4 \%, 2 \%$, and $3 \%$. The wall-normal position, $\eta_{0}$, of the symmetry point was determined by the location of the minimum of the sum of $\left[S(\eta) S\left(2 \eta_{0}-\eta\right)-S^{2}\left(\eta_{0}\right)\right]^{2}$, over all values of $\eta$ within the interval $\left[100 / h^{+}, 0.85\right]$, and by varying $\eta_{0}$ between 0.45 and 0.55 . The maximum relative deviation from the channel quarter-width is \pm 0.005 for the Reynolds numbers of Fig. 1 .

This symmetry property is perhaps more clearly highlighted by examining the derivative of $\log (S)$ with respect to $\eta$

$$
\frac{\mathrm{d} \ln S}{\mathrm{~d} \eta}=\frac{h^{+} S^{\prime+}}{S^{+}},
$$

where $S^{\prime}=\mathrm{d} S / \mathrm{d} y$, which, as shown in Fig. 2, has a symmetric profile about $\eta \approx 0.5$. We find best agreement for the 


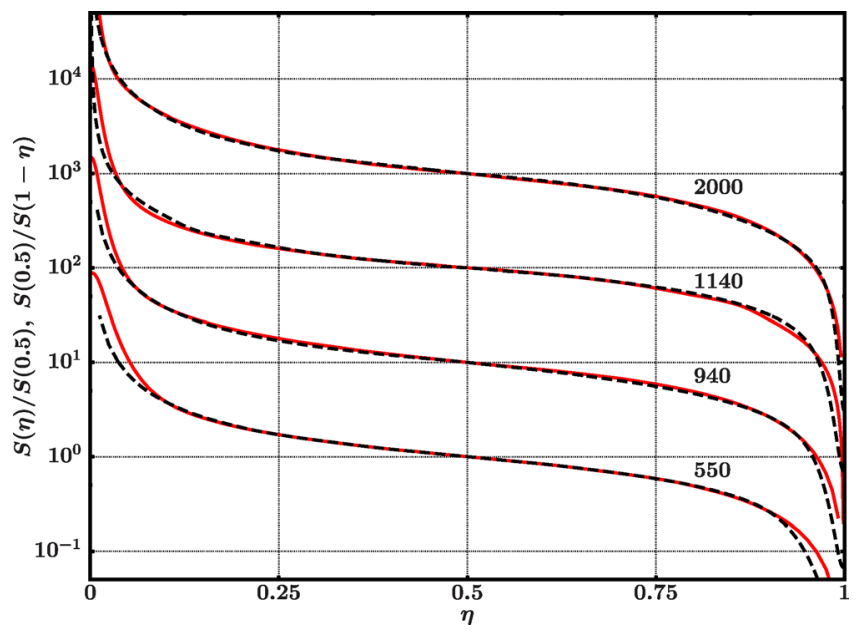

FIG. 1. (Color online) Mean velocity gradient $S(\eta)$ normalized by $S(0.5)$ for channel flow at $R_{\tau}=550,940$, and 2000 (Ref. 1) and pipe flow at $R_{\tau}=1140$ (Ref. 2). - (solid gray line), $S(\eta) / S(0.5) ;--$ (dashed black line), $S(0.5) /$ $S(1-\eta)$. The profiles are shifted upwards by a decade with increasing $R_{\tau}$.

profile at $R_{\tau}=550$, which is less noisy than the higher Reynolds number profiles where the observed scatter could be caused by a lack of convergence of the second derivative of the mean velocity. This figure also shows that the minimum value is Reynolds-number-independent and has a value of 1.6. It should be noted that the reciprocal of this quantity corresponds to the von Kármán mixing-length $\ell$ divided by $h^{3}$ Therefore, this length-scale is symmetric with respect to $\eta \approx 0.5$ and has a maximum of $\ell \approx 0.63 \mathrm{~h}$.

This symmetry property becomes more important in light of recent work of Mizuno and Jiménez, ${ }^{4}$ published during the revision of the present letter, who found that, in the overlap region of wall-bounded flows, the length scale based on the mean local shear

$$
\ell^{+}(\eta)=\frac{1}{S^{+}(\eta)}
$$

is more appropriate as a normalization length-scale than the classical mixing length proportional to $y$. According to

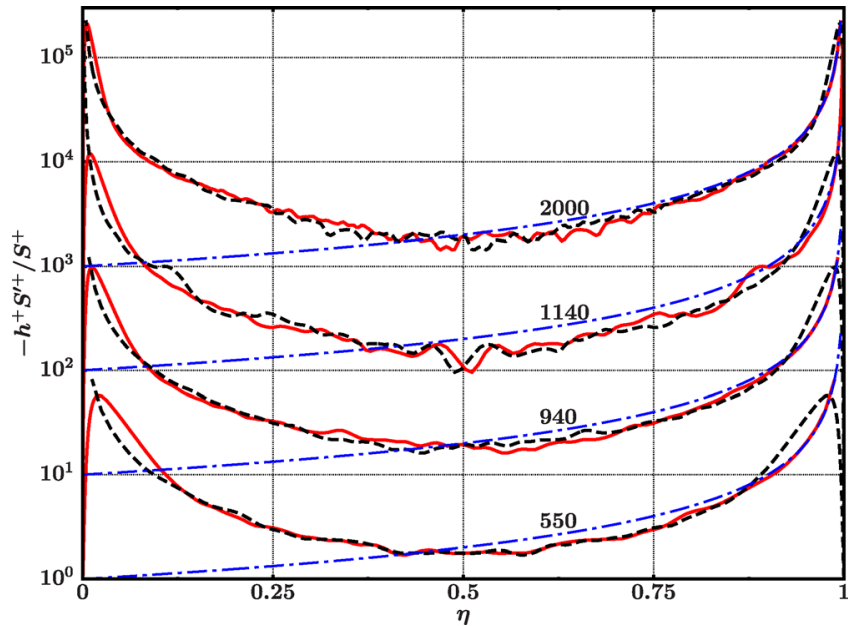

FIG. 2. (Color online) Profiles of the ratio of the viscous transport term $S^{\prime+}$ to the viscous stress $S^{+}$. — (solid gray line), $-h^{+} S^{\prime+} / S^{+}(\eta) ;--($ dashed black line $),-h^{+} S^{+} / S^{+}(1-\eta) ;-\cdot-($ dash-dotted black line $), 1 /(1-\eta)$. The profiles are shifted upwards by a decade with increasing $R_{\tau}$. relation (3), the local mean-shear based length should have the symmetry

$$
\frac{\ell(\eta)}{\ell(0.5)} \simeq \frac{\ell(0.5)}{\ell(1-\eta)} .
$$

In order to exploit the result given by relation (3), it is important to know how $S(0.5)$ varies with Reynolds number. Figure 3 shows the evolution of $S(0.5)$, normalized by the friction velocity $u_{\tau}$ and $h$, as a function of $R_{\tau}$ for channel and pipe flows. The DNS data for channel flow and $R_{\tau}>500$, which are free from low Reynolds number effects, ${ }^{5}$ give a constant value of $h^{+} S^{+}(0.5) \approx 6.3$. For pipe flow, the DNS data ${ }^{2}$ and the results of the super-pipe experiment ${ }^{6}$ show higher but constant values of about 7 at large Reynolds numbers. This difference suggests that the geometry affects the intensity of the mean velocity gradient more than its distribution. Note that, for the channel flow at $\eta \approx 0.5$, the viscous transport term, $-h^{+2} S^{\prime+}(0.5)$, has a value slightly higher than 10 , as shown in Fig. 4, where $-h^{+2} S^{\prime+}(\eta)$ is plotted against $\eta$ together with $-h^{+2} S^{\prime+}(1-\eta)$ obtained from relation (3)

$$
S^{\prime+}(1-\eta) \simeq S^{\prime+}(\eta)\left[\frac{S(0.5)}{S(\eta)}\right]^{2} .
$$

This figure not only confirms the result of Figs. 1 and 2 but it also shows that $-h^{+2} S^{\prime+}(\eta)$ has a minimum of about 10 , located at $\eta \approx 0.58$, and rises slowly to a Reynoldsnumber-independent centerline value of 16 , leading to the empirical relation

$$
-\frac{\mathrm{d} \overline{u v}^{+}}{\mathrm{d} y^{+}}(1) \simeq-\frac{1}{h^{+}}+\frac{16}{h^{+2}}
$$

at the center of the channel.

Another illustration of the usefulness of relation (3) are the implications for the eddy viscosity $\nu_{t}$, the ratio of turbulent and viscous stresses. We can express $\nu_{t}$ using relation (2), giving

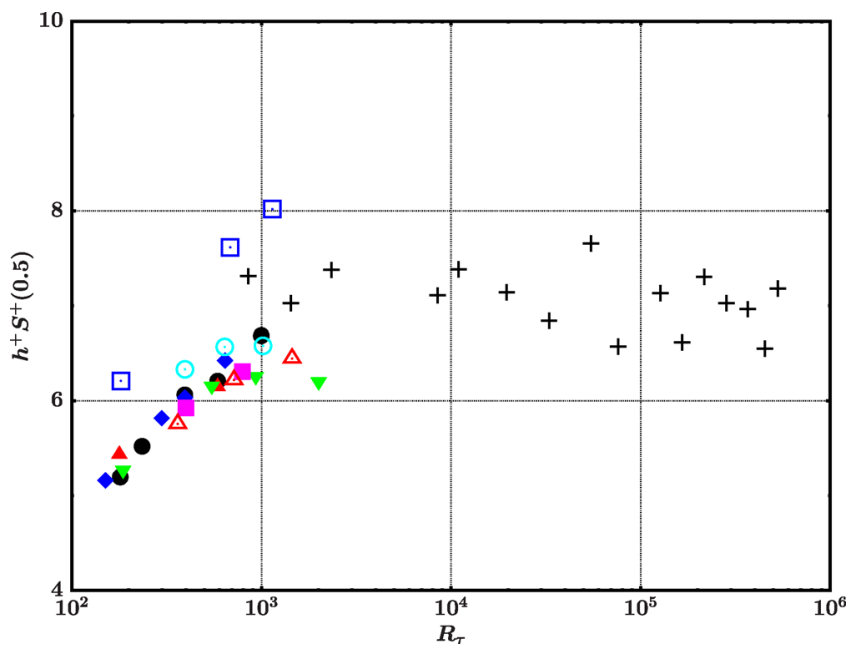

FIG. 3. (Color online) Evolution with the friction Reynolds number $R_{\tau}$ of $h^{+} S^{+}(0.5)$. DNS data of channel flow: •, Laadhari (Ref. 10); $\bullet$, Iwamoto et al. (Ref. 11); $\boldsymbol{\nabla}$, Jiménez and Hoyas (Refs. 1 and 12); $\boldsymbol{\Delta}$, Moser et al. (Ref. 5); $\mathbf{\square}$, Tanahashi et al. (Ref. 13); $\odot$, Abe et al. (Ref. 14); $\Delta$, Hu et al. (Ref. 9). DNS of pipe flow: $\square$, Wu and Moin (Ref. 2). Measurements in pipe flow: (+), Zagarola and Smits (Ref. 6). 


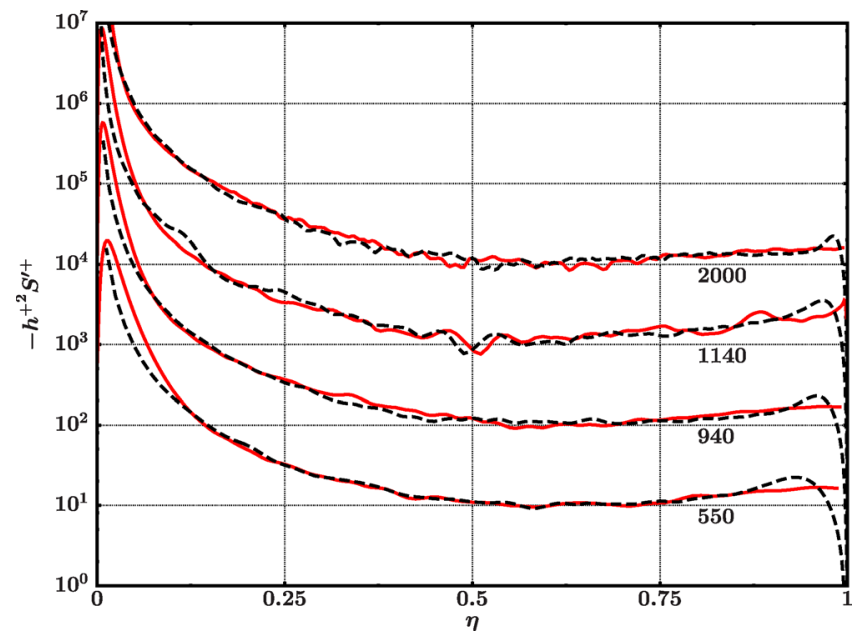

FIG. 4. (Color online) Profiles of the viscous transport term $-h^{+2} S^{\prime+}$ (solid gray line), $-h^{+2} S^{\prime+} ;--\left(\right.$ dashed black line), $-h^{+2} S^{\prime+}(1-\eta)$. The profiles are shifted upwards by a decade with increasing $R_{\tau}$.

$$
\nu_{t}^{+}(\eta)=-1+\frac{1-\eta}{S^{+}(\eta)} .
$$

Then, from Eq. (3), the eddy viscosities at $\eta$ and $1-\eta$ are related by

$$
\nu_{t}^{+}(1-\eta) \simeq-1+\frac{\eta(1-\eta)}{S^{+2}(0.5)\left[\nu_{t}^{+}(\eta)+1\right]} .
$$

The two expressions have been plotted in Fig. 5 for $\nu_{t}^{+} / h^{+}$as a function of $\eta$ for four different values of $R_{\tau}$; the curves collapse well, particularly for $R_{\tau}=550$ and 2000, everywhere we expect relation (3) to be applicable. It is worth noting that with the present results, the value of the eddy viscosity midway between the wall and the centerline is given by

$$
\nu_{t}^{+}(0.5) \simeq-1+\frac{1}{2 S^{+}(0.5)} \simeq-1+0.079 h^{+}
$$

for the channel flow and takes a lower value for the pipe flow due to the higher value of $S^{+}(0.5)$. It should be noted that the maximum of $\nu_{t}$ occurs when

$$
-h^{+} \frac{S^{\prime+}}{S^{+}}=\frac{1}{(1-\eta)},
$$

and for a logarithmic mean velocity profile, this relation leads to a peak location of $\nu_{t}$ at $\eta=0.5 .^{7}$ However, the two terms of Eq. (5), plotted in Fig. 2, intersect at a point located slightly nearer the wall than the channel quarter-width $(\eta \approx 0.45)$ leading to a peak value given by

$$
\nu_{t_{\max }}^{+} \simeq-1+0.080 h^{+} \text {. }
$$

In addition, the centerline value of $\nu_{t}^{+} / h^{+}$is constant, and this indicates that the mean velocity gradient is a function of $1-\eta$ near the center of the channel.

For illustrative purposes, the linear expression for the premultiplied mean velocity gradient, proposed by Jiménez and Moser [Ref. 8, Eq. (4.8)] for the region $0.15<\eta<0.5$ and $R_{\tau}=2000$, was used

$$
y^{+} S^{+}=\frac{1}{\kappa}+\frac{\beta}{h^{+}}+\alpha \eta
$$

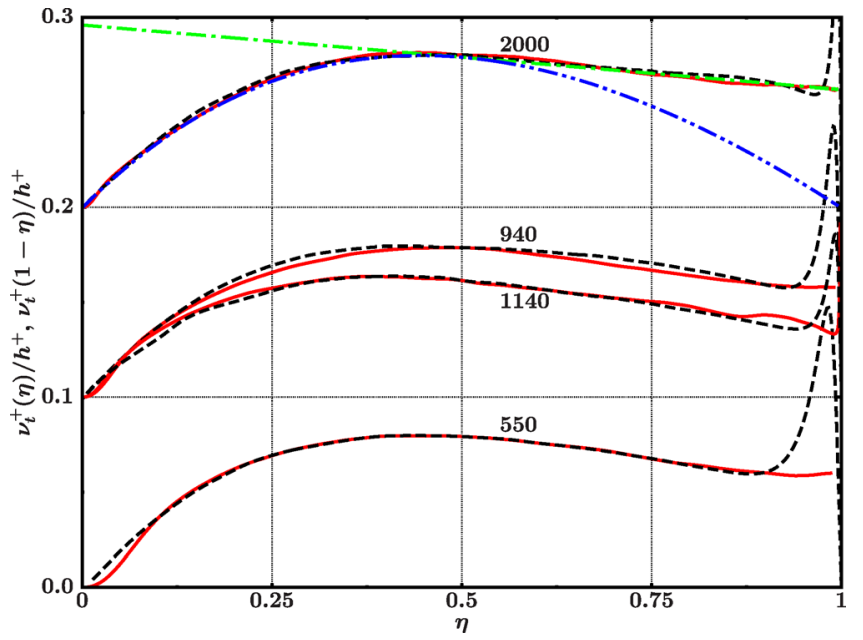

FIG. 5. (Color online) Profiles of the eddy viscosity $\nu_{t}$ normalized by $u_{\tau}$ and $h$. - (solid gray line), $\nu_{t}^{+} / h^{+}(\eta) ;--($ dashed black line $), \nu_{t}^{+} / h^{+}(1-\eta)$; - -. - (dash-double-dotted black line), relation (9); --- (dash-dotted gray line), relation (11). The profiles are shifted upwards by 0.1 with increasing $R_{\tau}$ except for $R_{\tau}=1140$ which has the same offset as $R_{\tau}=940$.

The left-hand side term is plotted in Fig. 6 against $\eta$ normalized on its value at $\eta=0.5$. Note that the linear behavior is also present in the DNS data of Hu et al. ${ }^{9}$ at $R_{\tau}=1440$. The best linear fit of the DNS data at $R_{\tau}=2000$ for $\eta$ within the range $0.15-0.5$ shows that the premultiplied mean gradient is described well by the following equation:

$$
2 \eta \frac{S(\eta)}{S(0.5)}=a(\eta-0.5)+1,
$$

with $a=3 / 7$. The right-hand side term and the ratio of the two terms of this equation are represented in Fig. 6 by the straight line and the plus symbol $(+)$, respectively. The maximum departure of the ratio from unity is of $\pm 1 \%$. The coefficients in relation (6) are then only a function of $h^{+} S^{+}(0.5)$, and the slope $a$,

$$
\begin{aligned}
\alpha & =h^{+} S^{+}(0.5) \frac{a}{2} \\
\frac{1}{\kappa}+\frac{\beta}{h^{+}} & =h^{+} S^{+}(0.5)\left(\frac{1}{2}-\frac{a}{4}\right) .
\end{aligned}
$$

The integration of $\mathrm{d} \bar{U}^{+} / \mathrm{d} \eta=h^{+} S^{+}(\eta)$ leads to the mean velocity profile

$$
\bar{U}^{+}=2.475 \ln y^{+}+1.35 \eta+4.85,
$$

which fits the DNS mean velocity data with a maximum relative departure of $0.1 \%$. The coefficient before the logarithmic term is very close to the reciprocal of the von Kármán constant. This result may just be fortuitous and must be considered as a particular case since it applies only for one Reynolds number case, and any attempt to extend it to large Reynolds numbers will require further investigation.

From relation (7), the mean velocity gradient is well characterized by

$$
\frac{S(\eta)}{S(0.5)}=\frac{a(\eta-0.5)+1}{2 \eta}
$$

and the eddy viscosity by the expression 


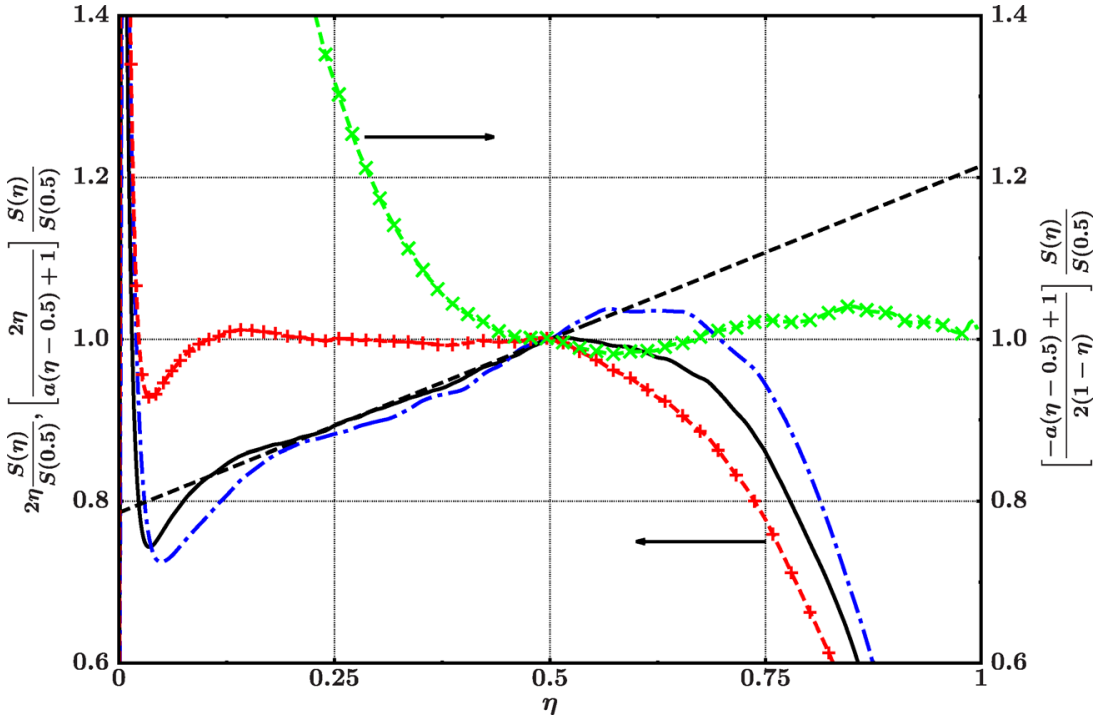

FIG. 6. (Color online) Normalized mean velocity gradient; $2 \eta \frac{S(\eta)}{S(0.5)}:-, R_{\tau}=2000$ [Jiménez and Hoyas (Ref. 1)]; - - - $R_{\tau}=1440$ [Hu et al. (Ref. 9)]; - -, $a(\eta-0.5)+1 ; \quad(-+-), \quad \frac{2 \eta}{a(\eta-0.5)+1 S(0.5)} ; \quad(-\times-)$, $\frac{-a(\eta-0.5)+1 S(\eta)}{2(1-\eta) \quad S(0.5)}$.

$$
\nu_{t}^{+}(\eta) \simeq-1+\frac{\eta(1-\eta)}{1.35 \eta+2.475} h^{+},
$$

which compares very well with the profile of $\nu_{t}$ at $R_{\tau}=2000$ of Fig. 5 when $\eta$ is within the range $0.15-0.5$. This expression is also in good agreement with the relationships for the maximum of the eddy viscosity and its value at $\eta=0.5$, provided above.

Combining relations (3) and (8), the mean velocity gradient for $\eta>0.5$ is given by

$$
\frac{S(\eta)}{S(0.5)}=\frac{2(1-\eta)}{-a(\eta-0.5)+1} .
$$

This is confirmed by the DNS data since the ratio of the two terms is fairly constant for $\eta>0.5$ as shown in Fig. 6. The departure from unity is of the same order as the statistical uncertainties in the premultiplied mean gradient at large $\eta$, evaluated by Jiménez and Moser $^{8}$ to be as high as 0.04 in the $R_{\tau}=2000$ case.

The corresponding eddy viscosity is then a linear function of $\eta$

$$
\nu_{t}^{+}(\eta) \simeq-1+\frac{-a(\eta-0.5)+1}{12.6} h^{+},
$$

and the resulting centerline value

$$
\nu_{t}^{+}(1) \simeq-1+0.062 h^{+}
$$

is well correlated with the DNS data as shown in Fig. 5.

The integration of relation (10) results in the mean velocity defect law,

$$
\frac{\bar{U}_{c}-\bar{U}(\eta)}{\overline{U_{c}}}=1.21(1-\eta)-2.219 \ln \frac{17-6 \eta}{11},
$$

with $\bar{U}_{c}$ the mean centerline velocity. The mean velocity data for $R_{\tau}=2000$ and $\eta>0.5$ agree well with this relation, with a relative discrepancy less than $0.1 \%$.

To conclude, in the present investigation, we discovered an apparent symmetry property of the mean velocity gradient in turbulent channel flow. The practical implications of this property have been illustrated by showing its relevance to the scaling of eddy viscosity and of the viscous term in the momentum balance. The validity of this property must be corroborated by further numerical simulations over a wider range of Reynolds numbers and accurate high precision measurements of the first and second mean velocity derivatives. A theoretical explanation of this result would no doubt improve our understanding of some of the fundamental mechanisms involved in wall bounded flows.

This work was granted access to the HPC resources of CINES under the allocation 2011-x2011026387 made by GENCI (Grand Equipement National de Calcul Intensif).

${ }^{1}$ J. Jiménez and S. Hoyas, "Turbulent fluctuations above the buffer layer of wall-bounded flows,” J. Fluid Mech. 611, 215 (2008).

${ }^{2} \mathrm{X}$. Wu and P. Moin, "A direct numerical simulation study on the mean velocity characteristics in turbulent pipe flow," J. Fluid Mech. 608, 81 (2008).

${ }^{3}$ T. Von Kármán, “Turbulence and skin friction,” J. Aeronaut. Sci. 1, 1 (1934), available at http://pdf.aiaa.org/jaPreview/PSJA/1934/PVJAPRE5.pdf.

${ }^{4}$ Y. Mizuno and J. Jiménez, "Mean velocity and length-scales in the overlap region of wall-bounded turbulent flows," Phys. Fluids 23, 085112 (2011).

${ }^{5}$ R. D. Moser, J. Kim, and N. N. Mansour, "Direct numerical simulation of turbulent channel flow up to $R e_{\tau}=590$," Phys. Fluids 11, 943 (1999).

${ }^{6}$ M. V. Zagarola and A. J. Smits, "Mean-flow scaling of turbulent pipe flow," J. Fluid Mech. 373, 33 (1998).

${ }^{7}$ J. Neumann, "Location of the eddy viscosity maximum in smooth pipe flow," Phys. Fluids 22, 1405 (1979).

${ }^{8}$ J. Jiménez and R. D. Moser, "What are we learning from simulating wall turbulence?,” Philos. Trans. R Soc. London, Ser. A 365, 715 (2007).

${ }^{9}$ Z. W. Hu, C. L. Morfey, and N. D. Sandham, "Wall pressure and shear stress spectra from direct simulations of channel flow," AIAA J. 44, 1541 (2006).

${ }^{10} \mathrm{~F}$. Laadhari, "Reynolds number effect on the dissipation function in wallbounded flows," Phys. Fluids 19, 038101 (2007).

${ }^{11}$ K. Iwamoto, Y. Suzuki, and N. Kasagi, "Reynolds number effect on wall turbulence: Toward effective feedback control," Int. J. Heat Fluid Flow 23, 678 (2002).

${ }^{12} \mathrm{~S}$. Hoyas and J. Jiménez, "Scaling of the velocity fluctuations in turbulent channels up to $R e_{\tau}=2003$," Phys. Fluids 18, 011702 (2006).

${ }^{13}$ M. Tanahashi, S.-J. Kangand, T. Miyamoto, S. Shiokawa, and T. Miyauchi, "Scaling law of fine scale eddies in turbulent channel flows up to $R e_{\tau}=800$," Int. J. Heat Fluid Flow 25, 331 (2004).

${ }^{14}$ H. Abe, H. Kawamura, and Y. Matsuo, "Surface heat-flux fluctuations in a turbulent channel flow up to $R e_{\tau}=1020$ with $\operatorname{Pr}=0.025$ and 0.71 ," Int. J. Heat Fluid Flow 25, 404 (2004). 